\title{
Quantized Fields Propagating in Plane-Wave Spacetimes
}

\author{
G. W. Gibbons \\ Department of Applied Mathematics and Theoretical Physics, \\ University of Cambridge, Cambridge CB3 9EW, U.K.
}

\begin{abstract}
This paper contains an account of the interaction of a quantized massive scalar field with the classical $c$ number gravitational field of a plane sandwich wave of arbitrary profile and polarization. It is shown that the time varying gravitational field of the wave produces no particles and the Feynman propagator for the problem is calculated exactly. This is used to show that any reasonable regularization of the vacuum expectation value of the energy momentum tensor of the field must vanish. This means that a gravitational wave far from its source will propagate without hindrance by quantum effects.
\end{abstract}

\section{Introduction}

Recently there has been some interest in a class of problems in which one treats a quantized field propagating on a classical, $c$-number, background gravitational field [1-4]. This work has encountered two sorts of difficulties:

(1) The definition of no particle states.

(2) The definition of a suitable energy momentum tensor operator $\boldsymbol{T}_{\mu \nu}$.

The first difficulty can be circumverted to some extent when the spacetime is asymptotically flat in some sense and interesting results have been obtained [1-3]. The second difficulty remains and becomes especially urgent if one seeks to determine the back reaction of the quantum field on the geometry in a HartreeFock approximation of the form

$$
R_{\mu \nu}-\frac{1}{2} g_{\mu \nu} R=8 \pi\left\langle\psi\left|\boldsymbol{T}_{\mu \nu}\right| \psi\right\rangle
$$

where $|\psi\rangle$ is some constant Heisenberg state vector - for example the initial no particle or vacuum state $\left|0_{-}\right\rangle$. An especially interesting question is will $\left\langle 0_{-}\left|\boldsymbol{T}_{\mu \nu}\right| 0_{-}\right\rangle$obey the various energy conditions used in the Singularity Theorems or other global results in classical general relativity [6]. The generally expected answer seems to be "no" but no exact calculations have been performed.

This paper contains an exact calculation of $\left\langle 0_{-}\left|\boldsymbol{T}_{\mu \nu}\right| 0_{-}\right\rangle$for the case of a plane sandwich wave of arbitrary profile and polarization in the case that the quantum field is spinless. The main result is that in such a spacetime no particles are produced by the time varying gravitational field and all reasonable suggestions for regularizing the divergent $\left\langle 0_{-}\left|\boldsymbol{T}_{\mu \nu}\right| 0_{-}\right\rangle$give a vanishing result. This shows 
that a gravitational wave far from its source will propagate without hindrance from quantum effects and is analogous to the corresponding result for electromagnetic waves proved by Schwinger [7]. It unfortunately gives no information on the energy conditions but it seems to be of interest as an illustration of the general formalism and it may provide some ideas for the general problem.

The paper is divided into four sections. In 1 the general formalism developed in a previous paper [3] is slightly extended, various propagators are defined and it is shown how they may be used to obtain $\boldsymbol{T}_{\mu \nu}$ and various approaches to its regularization are described. Section 2 contains an outline of the properties of plane sandwich waves which are required. Section 3 contains a proof that the wave creates no particles and the exact calculation of the (unique) Feynmann propagator. The relationship of these results to the problem of back scattering is discussed. In Section 4 a comparison is made with some results of deWitt and the paper finishes with a short conclusion.

\section{General Formalism}

In this section we wish to outline some methods for obtaining the vacuum expectation value of the energy momentum tensor $\boldsymbol{T}_{\mu \nu}$ of a quantum field $\boldsymbol{\phi}$ propagating on a classical $c$ number gravitational background. For simplicity we restrict attention to a scalar (not necessarily hermitian) field. The gravitational field is described by a spacetime manifold $\left\{\mathscr{M}, g_{\mu \nu}\right\}$ which, in view of the general discussion in [3], we assume to possess a Cauchy surface. This will ensure that there is a well defined time ordering $T$ of events $x, x^{\prime}, \ldots, x^{\prime \prime} \ldots$ such that for position dependent operators $A(x), A\left(x^{\prime}\right) \ldots A\left(x^{\prime \prime}\right) \ldots$ which commute at spacelike seperations $T\left\{\boldsymbol{A}(x) \boldsymbol{A}^{\prime}\left(x^{\prime}\right) \ldots \boldsymbol{A}^{\prime \prime}\left(x^{\prime \prime}\right) \ldots\right\}$ is the product of these operatiors arranged in chronological order, the latest occurring on the left. In general the product diverges if any two arguments can be joined by a null geodesic or coincide and so we shall not define $T$ in that case. Time ordering merely requires the chronology (no closed timelike lines) but this is guaranteed if a Cauchy surface exists. In the detailed case we consider later (plane waves) Cauchy surfaces do not exist but the chronology condition does hold. We shall use an heuristic argument to include it in the general formulation.

We shall also assume that $\mathscr{M}$ contains two regions one to the past and one to the future $\mathscr{M}^{-}$and $\mathscr{M}^{+}$which are sufficiently flat that one can define ingoing and outgoing vacuum states $\left|0_{-}\right\rangle$and $\left|0_{+}\right\rangle$associated with them. These will be defined using bases $\left\{p_{i}, n_{i}\right\}=B_{-}$and $\left\{p_{i}^{\prime}, n_{i}^{\prime}\right\}=B_{+}$of solutions of the wave equation satisfield by $\phi$ - the Klein Gordon equation. Some of our calculations could be carried out in any basis and the result will be referred to as Bogoliubov or basis independent. We shall say that two bases $B$ and $B^{\prime}$ are connected by a non-mixing Bogoliubov transformation if the scalar products:

$\left(p_{i}, n_{j}^{\prime}\right)$ and $\left(n_{i}, p_{j}^{\prime}\right)$ vanish for all the indicated elements of $B$ and $B^{\prime}$. The condition for no particle creation is that $B_{-}$and $B_{+}$to be connected by a nonmixing Bogoliubov transformation.

The commutation relations of the field $\phi$ with its hermitian congugate $\phi^{\dagger}$ are defined by

$$
\left[\phi(x), \phi^{\dagger}\left(x^{\prime}\right)\right]=i \mathscr{G}\left(x, x^{\prime}\right)
$$


where $\mathscr{G}\left(x, x^{\prime}\right)$ is a certain (Bogoliubov invariant) solution of the homogeneous Klein-Gordon equation, which was defined in [3].

In terms of an arbitrary basis it has the form:

$$
i \mathscr{G}\left(x, x^{\prime}\right)=\sum_{i} p_{i}(x) \bar{p}_{i}\left(x^{\prime}\right)-n_{i}(x) \bar{n}_{i}\left(x^{\prime}\right) .
$$

From $\mathscr{G}\left(x, x^{\prime}\right)$ we may define the advanced and retarded Green's functions $\mathscr{G}^{\mathrm{adv}}\left(x, x^{\prime}\right)$ and $\mathscr{G}^{\mathrm{ret}}\left(x, x^{\prime}\right)$ which obey the inhomogeneous equation: by

$$
\left(\square^{2}+m^{2}\right) \mathscr{G}^{\mathrm{ret}}\left(x, x^{\prime}\right)=\delta\left(x, x^{\prime}\right)=\left(\square^{2}+m^{2}\right) \mathscr{G}^{\mathrm{adv}}\left(x, x^{\prime}\right)
$$

$$
\mathscr{G}\left(x, x^{\prime}\right)=\mathscr{G}^{\mathrm{adv}}\left(x, x^{\prime}\right)-\mathscr{G}^{\mathrm{ret}}\left(x, x^{\prime}\right)
$$

and the condition that $\mathscr{G}^{\text {adv }}\left(x, x^{\prime}\right)\left(\mathscr{G}^{\text {ret }}\left(x, x^{\prime}\right)\right)$ vanish if $x^{\prime}$ chronologically precedes (antedates) $x . \delta\left(x, x^{\prime}\right)$ is a four dimensional delta function. Strictly speaking we are now regarding $\hat{\phi}(x)$ as an operator valued distribution. A third Bogoliubov invariant Green's function is

$$
\overline{\mathscr{G}}\left(x, x^{\prime}\right)=\frac{1}{2}\left\{\mathscr{G}^{\mathrm{ret}}\left(x, x^{\prime}\right)+\mathscr{G}^{\mathrm{adv}}\left(x, x^{\prime}\right)\right\}
$$

Now given a "vacuum state" $|0\rangle$ defined by a basis $B$ it is possible to define a Feynman Propagator as

$$
\begin{aligned}
\left\langle 0\left|T\left\{\phi(x) \phi^{\dagger}\left(x^{\prime}\right)\right\}\right| 0\right\rangle & =-i \mathscr{G}_{F}\left(x, x^{\prime}\right) \\
& =\sum_{i} p_{i}(x) \bar{p}_{i}\left(x^{\prime}\right)=-i \mathscr{G}^{(+)}\left(x, x^{\prime}\right) \quad \text { if } \quad x^{\prime} \text { precedes } x \\
& =\sum_{i} n_{i}(x) \bar{n}_{i}\left(x^{\prime}\right)=+i \mathscr{G}^{(-)}\left(x, x^{\prime}\right) \quad \text { if } x \text { precedes } x^{\prime} .
\end{aligned}
$$

$\mathscr{G}_{F}, \mathscr{G}^{(-)}$and $\mathscr{G}^{(+)}$are basis dependent but they are invariant under nonmixing Bogoliubov transformations. $\mathscr{G}_{F}-$ which obeys the inhomogeneous Klein-Gordon Eq. (1.3) has the property of propagating "particles" to the future and "antiparticles" to the past where particles are the excitations corresponding to the $p_{i}$ and need not be "real" particles which are only defined in the asymptotic regions. Given two such asymptotic regions there is an alternative and in general inequivalent definition of a Feynman Propagater, which we shall write as $\mathscr{G}_{\infty}\left(x, x^{\prime}\right)$ by the equation

$$
-i \mathscr{G}_{\infty}\left(x, x^{\prime}\right)=\left\langle 0+\left|T\left\{\phi(x) \phi^{\dagger}\left(x^{\prime}\right)\right\}\right| 0_{-}\right\rangle\left\langle\left\langle 0_{+} \mid 0_{-}\right\rangle\right.
$$

Some properties of these propagatiors are tabulated in Table 1.

It now remains to note that

$$
2 \boldsymbol{T}_{\mu \nu}(x)=\nabla_{\mu} \boldsymbol{\phi}(x) \nabla_{\mu} \boldsymbol{\phi}^{\dagger}(x)-\frac{1}{2} g_{\mu \nu}(x)\left\{\nabla_{\alpha} \boldsymbol{\phi}(x) \nabla^{\alpha} \boldsymbol{\phi}^{\dagger}(x)-m^{2} \boldsymbol{\phi}(x) \boldsymbol{\phi}^{\dagger}(x)\right\}+\text { h.c. }
$$

may be written as

$$
\boldsymbol{T}_{\mu v}(x)=\operatorname{Lt}_{x \rightarrow x^{\prime}} \mathscr{T}_{\mu v^{\prime}} \frac{1}{2}\left\{\boldsymbol{\phi}(x) \boldsymbol{\phi}^{\dagger}\left(x^{\prime}\right)+\boldsymbol{\phi}^{\dagger}(x)+\boldsymbol{\phi}^{\dagger}(x) \boldsymbol{\phi}\left(x^{\prime}\right)\right\},
$$

where

$$
\begin{aligned}
\mathscr{T}_{\mu \nu^{\prime}} & =\nabla_{\mu} \nabla_{v^{\prime}}-\frac{1}{2} g_{\mu v^{\prime}}\left(x, x^{\prime}\right)\left\{g^{\alpha \alpha^{\prime}}\left(x, x^{\prime}\right) \nabla_{\alpha} \nabla_{\alpha^{\prime}}-m^{2}\right\} \\
\nabla_{\mu} & =\partial / \partial x^{\mu} ; \quad \nabla_{v^{\prime}}=\partial / \partial x^{\prime v}
\end{aligned}
$$

and $g_{\mu v^{\prime}}\left(x, x^{\prime}\right)$ is the 2 point vector of parallel transport [11]. 
Table 1

\begin{tabular}{|c|c|c|c|c|c|}
\hline Name & Symbol & $\begin{array}{l}\text { Bogoliubov } \\
\text { invariant? }\end{array}$ & $\begin{array}{l}\text { Satisfy what } \\
\text { equation }\end{array}$ & $\begin{array}{l}\text { Support } \\
(\text { for fixed } x \text { ) }\end{array}$ & Symmetries \\
\hline $\begin{array}{l}\text { Jordan-Paulı } \\
\text { function }\end{array}$ & $\mathscr{G}\left(x, x^{\prime}\right)$ & Yes & Homogeneous & $\begin{array}{l}\text { Interior and closure } \\
\text { of light cone }\end{array}$ & $\mathscr{G}\left(x, x^{\prime}\right)=-\mathscr{G}\left(x^{\prime}, x\right)$ \\
\hline $\begin{array}{l}\text { Retarded } \\
\text { Green's } \\
\text { function }\end{array}$ & $\mathscr{G}^{\mathrm{ret}}\left(x, x^{\prime}\right)$ & Yes & Inhomogeneous & $\begin{array}{l}\text { Interior and closure } \\
\text { of past light cone }\end{array}$ & $\mathscr{G}^{\mathrm{adv}}\left(x, x^{\prime}\right)=\mathscr{G}^{\mathrm{ret}}\left(x^{\prime}, x\right)$ \\
\hline \multirow[t]{3}{*}{$\begin{array}{l}\text { Advanced } \\
\text { Green's } \\
\text { function }\end{array}$} & $\mathscr{G}^{\mathrm{adv}}\left(x, x^{\prime}\right)$ & Yes & Inhomogeneous & $\begin{array}{l}\text { Interior and closure } \\
\text { of future light cone }\end{array}$ & \\
\hline & $\mathscr{G}^{(+)}\left(x, x^{\prime}\right)$ & No & Homogeneous & $\mathscr{U}$ & $\overline{\mathscr{G}^{(+)}\left(x, x^{\prime}\right)}=-\mathscr{G}^{(+)}\left(x^{\prime}, x\right)$ \\
\hline & $\mathscr{G}^{(-)}\left(x, x^{\prime}\right)$ & No & Homogeneous & U & $\overline{\mathscr{G}^{(-)}\left(x, x^{\prime}\right)}=-\mathscr{G}^{(-)}\left(x^{\prime}, x\right)$ \\
\hline Feynman & $\mathscr{G}_{F}\left(x, x^{\prime}\right)$ & No & Inhomogeneous & U & $\overline{\mathscr{G}_{F}\left(x, x^{\prime}\right)}=-\mathscr{G}_{F}\left(x^{\prime}, x\right)$ \\
\hline Propagator & $\mathscr{G}_{\infty}\left(x, x^{\prime}\right)$ & No & Inhomogeneous & $\mathscr{U}$ & \\
\hline
\end{tabular}

If no electromagnetic field is present we may set $n_{1}=\bar{p}_{1} \cdot \mathscr{G}\left(x, x^{\prime}\right)$ becomes real, $\mathscr{G}\left(x, x^{\prime}\right)$ is pure imaginary and hence $\mathscr{G}_{F}\left(x, x^{\prime}\right)=\mathscr{G}_{F}\left(x^{\prime}, x\right) \cdot \overline{\mathscr{G}^{-}\left(x, x^{\prime}\right)}=\mathscr{G}^{(+)}\left(x, x^{\prime}\right)$ and $\mathscr{G}^{(+)}\left(x, x^{\prime}\right)=-\mathscr{G}^{(-)}\left(x, x^{\prime}\right)$.

Thus

$$
\begin{aligned}
\left\langle 0\left|\boldsymbol{T}_{\mu \nu}\right| 0\right\rangle & =-i / 2 \operatorname{Lt}_{x \rightarrow x^{\prime}} \mathscr{T}_{\mu v^{\prime}}\left\{\mathscr{G}^{(+)}\left(x, x^{\prime}\right)-\mathscr{G}^{(-)}\left(x, x^{\prime}\right)\right\} \\
& =-i \operatorname{Lt}_{x \rightarrow x^{\prime}} \mathscr{T}_{\mu \nu^{\prime}} \mathscr{G}_{F}\left(x, x^{\prime}\right) .
\end{aligned}
$$

Where to ensure the validity of the transition from (1.12) to (1.13) we must take the average of the limits going from the future and the past [8]. Similar expressions may be written for the vacuum current. Thus we see that $\mathscr{G}_{F}\left(x, x^{\prime}\right)$ contains all the information we need and since it is a scalar quantity we expect that it will be easier to calculate $\left\langle 0_{-}\left|\boldsymbol{T}_{\mu v}\right| 0_{-}\right\rangle$using it than to perform a mode by mode addition which involves calculating 10 quantities for each mode and then adding. This idea is due to Schwinger $[7,8]$. The difficulty is that the resultant limit diverges, and may still depend upon the direction in which it is taken within the light cone. Indeed (1.9) may not be equivalent to (1.12). However, since in any case (1.9) is poorly defined we prefer to work with (1.13) as our basic "definition" of $\boldsymbol{T}_{\mu v}$. In order to extract a finite result a number of approaches suggest themselves.

(1) "Normal ordering" using some arbitrary basis. If we use $\left|0_{-}\right\rangle$we get identically zero. If we use $\left|0_{+}\right\rangle$we shall have some incoming energy flux (possibly infinite) in the infinite past. Related to this idea is that of using $\left\langle 0_{+}\left|\boldsymbol{T}_{\mu \nu}\right| 0_{+}\right\rangle_{-}$ $\left\langle 0_{-}\left|\boldsymbol{T}_{u \nu}\right| 0_{-}\right\rangle$which suffers from the same problems.

(2) "Adiabatic Regularization" [4] in which one performs a mode by mode subtraction of the value of $\boldsymbol{T}_{\mu \nu}$ in the limit that the gravitational field does not change with time - this is not covariant nor very well defined for more general contexts than the specific example in which it has been used.

(3) Pauli-Villars type regularization $[9,10]$. This was introduced into gravitational problems by Zel'dovich and Starobinsky [10]. In their sense it consists of subtracting from $\boldsymbol{T}_{\mu \nu}$ the $\boldsymbol{T}_{\mu \nu}$ of some auxillary fields with masses $M_{i}$ and amplitudes $\sqrt{c_{i}}$ and choosing $M_{i}$ and $c_{i}$ such that the divergences disappear 
and then taking the limit as $M_{i} \rightarrow \infty$. From the above this is entirely equivalent to regularization of the propagater $\mathscr{G}_{F}\left(x, x^{\prime}\right)$ in the Pauli-Villars fashion [9] and then taking the limit in (1.13). A simple version of this would be to subtract from $\mathscr{G}_{F}\left(x, x^{\prime}\right)$ its value in field free flat space expressed in some invariant way. In flat space $\mathscr{G}_{F}$ is given by

$$
D_{F}=-\left(16 \pi^{2}\right)^{-1} \int_{0}^{\infty} e^{i m^{2} / s} e^{i \Omega s / 2} d s,
$$

where $\Omega=\frac{1}{2} \eta_{\mu \nu} x^{\mu} x^{\nu}$. If we replace $\Omega$ by $\Omega\left(x, x^{\prime}\right)$ the "world function" [11] we have an invariantly defined biscalar to subtract from $\mathscr{G}_{F}\left(x, x^{\prime}\right)$ before we take the limit in (1.13). It will turn out to be more convenient and equally valid to subtract from $\mathscr{G}_{F}\left(x, x^{\prime}\right)$ the quantity:

$$
-\left(16 \pi^{2}\right)^{-1} \sqrt{\Delta} \int_{0}^{\infty} \exp i\left\{m^{2} / s+\Omega s / 2\right\} d s,
$$

where

$$
\Delta\left(x, x^{\prime}\right)=\left[g(x) g\left(x^{\prime}\right)\right]^{-\frac{1}{2}} \operatorname{det}\left|\nabla_{\alpha} \nabla_{\alpha^{\prime}} \Omega\right|
$$

is the Van Vleck determinant which occurs in problems involving two point tensors and Green's Functions [11].

In the plane wave spacetimes it is shown in Section 3 that $\mathscr{G}_{F}\left(x, x^{\prime}\right)$ is precisely of this form and so the regularized propagator vanishes and $\left\langle 0_{-}\left|\boldsymbol{T}_{\mu \nu}\right| 0_{-}\right\rangle=0$. However, in a more general spacetime there might be further divergences and indeed deWitt and Utiyama $[12,13]$ have argued that this is the case. This would mean that more than one auxillary mass is needed to perform the Pauli-Villars regularization. DeWitt's approach is described in more detail in Section 4.

\section{The Spacetime}

We wish to consider the spacetime $\left\{\mathscr{M}, g_{\mu \nu}\right\}$ with the following structure:-

(1) The manifold is $R^{4}$.

(2) An atlas for is provided by the single co-ordinate chart consisting of co-ordinates $\left\{U, V, X^{1}, X^{2}\right\}$ which run over the reals.

(3) In these co-ordinates the metric is given by:

$$
d s^{2}=d U d V+H_{i j}(U) X^{i} X^{j} d U^{2}-d X^{i} d X^{i}
$$

$i=1,2$ and $H_{i j}(U)$ are $C^{1}$ functions of $U$.

$\left\{\mathscr{M}, g_{\mu \nu}\right\}$ is geodesically complete and satisfies the chronology condition. In these co-ordinates the only non vanishing components of the Rieman tensor are:

$$
R_{U j U}^{i}=H_{i j}(U) .
$$

$\left\{\mathscr{M}, g_{\mu \nu}\right\}$ also has the property that all scalar invariants formed from $g_{\mu \nu}$ and $R_{\mu v \sigma \rho}$ and its covariant derivatives vanish identically.

Although it is not obvious from (2.1) $g_{\mu \nu}$ is invariant under a five parameter group of diffeomorphisms $G_{5}$ which contains an Abelian subgroup $G_{3}$ which act transitively on the null surfaces $U=$ constant, a one dimensional subgroup of 
which acts along the null generators [14]. In order to exhibit the $G_{3}$ explicitly we introduce new co-ordinate charts $\left\{u, v, x^{1}, x^{2}\right\}$ related to the old ones by:

$$
\begin{aligned}
U & =u \\
V & =v+\frac{1}{2} a_{i j}(u) x^{i} x^{j} \\
X^{i} & =P_{j}^{i}(u) x^{j},
\end{aligned}
$$

where

$$
\begin{aligned}
P_{i}^{m}(u) P_{j}^{m}(u) & =a_{i j}(u) \\
\ddot{P}_{j}^{m}(u) & =H_{m r} P_{j}^{r}(u) \\
\frac{\partial\left(u, v, x^{1}, x^{2}\right)}{\partial\left(U, V, X^{1}, X^{2}\right)} & =a=\operatorname{det}\left|a_{i j}(u)\right|
\end{aligned}
$$

and which takes the metric to the form:

$$
d s^{2}=d u d v-a_{i j}(u) d x^{i} d x^{j}
$$

It is clear that there are many such charts, each specified by giving $a_{i j}$ and $a_{i j}$ for some value of $u$. However, each one becomes singular for some value of $u$ as may be seen as follows. By manipulating (2.4) one may obtain

$$
\frac{d^{2} \sqrt{a}}{d u^{2}} \leqq \sqrt{a} H_{m m}
$$

This is sufficient to show that $a$ vanishes for at least one value of $u$ since by the weak energy condition $H_{m m} \leqq 0$ (strictly speaking if $H_{m m}=0$ we cannot use (2.6) directly to prove this but it remains true). Thus if we are to use the group co-ordinates we shall need at least two charts.

A sandwich wave is of the form (2.1) with $H_{i j}(U)=0$ for $U \leqq U_{1}$ and $U \geqq U_{2}$. It thus consists of two flat regions $\mathscr{M}^{-}$and $\mathscr{M}^{+}$separated from a non flat region $\mathscr{M}^{0}$ by two null surfaces. $\mathscr{M}^{+}$and $\mathscr{M}^{-}$have associated with them two charts such that $a_{i j}(u)=\delta_{i j}$ and $a_{i j}(u)=0$ for $U \leqq U_{1}$ and $U \geqq U_{2}$ respectively.

The value of the group co-ordinates is that solving for geodesics and solutions of the Klein-Gordon equation is especially simple using them. In particular the world function and the Van Vleck determinant are easily seen to be given by

$$
\begin{aligned}
& \Omega=\left(u-u^{\prime}\right)\left(v-v^{\prime}\right)-B_{i j}\left(u, u^{i}\right)\left(x^{i}-x^{\prime i}\right)\left(x^{j}-x^{\prime j}\right), \\
& \Delta=\left\{a(u) a\left(u^{\prime}\right)\right\}^{-\frac{1}{2}} \operatorname{det}\left|B_{i j}\left(u, u^{\prime}\right)\right|,
\end{aligned}
$$

where

$$
\begin{aligned}
\left(u-u^{\prime}\right)^{-1} B_{i j} \int_{u^{\prime}}^{u} d u^{\prime \prime} a^{j k}\left(u^{\prime \prime}\right) & =\delta_{i}^{k} \\
a^{i j}(u) a_{j k}(u) & =\delta_{i}^{k} .
\end{aligned}
$$

$\left\{\mathscr{M}, g_{\mu \nu}\right\}$ possesses the remarkable property of containing no Cauchy surface [15]. However, an examination of the geodesics shows that only those geodesics travelling along lines of constant $U$ fail to meet the null surface $U=$ constant. As far as particle creation is concerned we are interested in the effect of the wave on "virtual" 
Fig. 1a. A sandwich wave. The diagram represents the $U, V$ plane for fixed $X^{1}$ and $X^{2}$. The dotted line is a typical null geodisic which does not intersect all $U=$ constant surfaces

Fig. 1b. Schematic Penrose diagram of an asymptotically flat spacetime with a bounded source which radiates for a finite time (ignoring tails). Near $\mathscr{I}^{+}$the geometry may be approximated by a plane wave. The dotted line represents a null geodesic from $\mathscr{I}^{+}$passing through the origin and intersecting $\mathscr{I}^{-}$. $\mathscr{I}^{-} \cup I^{-}$form a Cauchy surface for the problem. To calculate particle production by the wave the details of the source need not be known and one may use the geometry of Fig. 1a
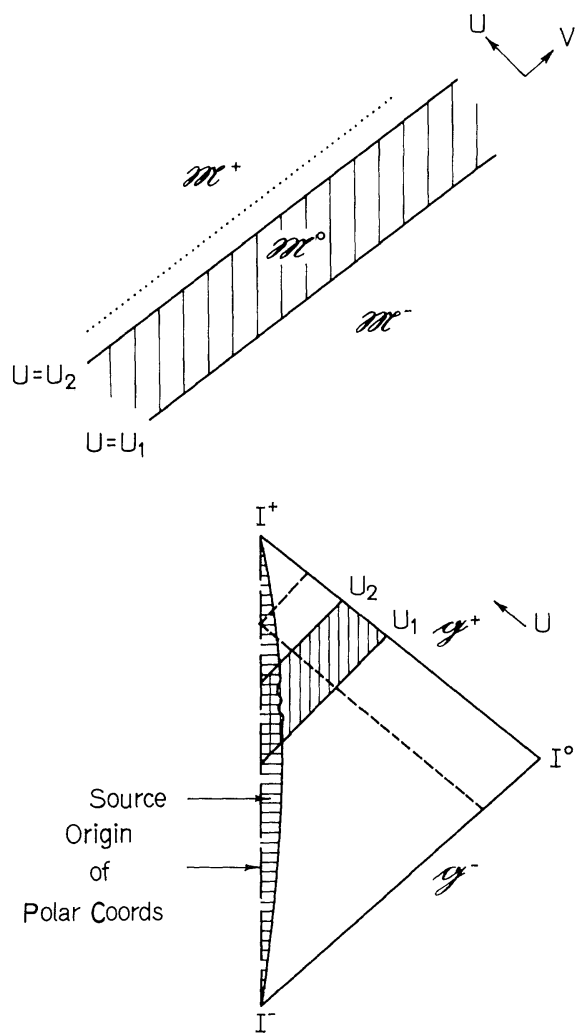

particles which pass through the wavefront. In a realistic example information travelling parallel to the wavefront would come from a source (see Fig. 1). Thus for finding the behaviour in $\mathscr{M}^{+}$due to data on $\mathscr{M}^{-}$it is sufficient to take as substitute Cauchy surfaces the null surfaces $U=$ constant.

\section{Calculation of Bogoliubov Coefficients and Propagators}

In order to define a basis of solutions in $\mathscr{M}^{-}$or $\mathscr{M}^{+}$we introduce in each a chart of group co-ordinates using the initial conditions $a_{i j}=\delta_{i j}, a_{i j}^{i}=0$ in $\mathscr{M}^{-}$or $\mathscr{M}^{+}$ respectively and consider the functions, which are solutions of the Klein-Gordon equation

$$
\begin{aligned}
& P_{i}=a(u)^{-\frac{1}{2}} \exp i\left\{a_{i} x^{i}+c v+\psi(u)\right\} \\
& c>0 ; \quad a_{1}, a_{2} \in R \\
& n_{i}=a(u)^{-\frac{1}{2}} \exp i\left\{a_{i} x^{i}+c v+\psi(u)\right\} \\
& c<0 ; \quad a_{1}, a_{2} \in R
\end{aligned}
$$

with

$$
\dot{\psi}(u)=\frac{a^{i j}(u) a_{i} a_{j}+m^{2}}{4 c} .
$$


Then

$$
\begin{aligned}
& \left(P_{i}, P_{j}\right)=\delta\left(c-c^{\prime}\right) \delta\left(a_{1}-a_{1}^{\prime}\right) \delta\left(a_{2}-a_{2}^{\prime}\right)(2 \pi)^{3} \\
& \left(P_{i}, n_{j}\right)=0 \\
& \left(n_{i}, n_{j}\right)=c \delta\left(c-c^{\prime}\right) \delta\left(a_{1}-a_{1}^{\prime}\right) \delta\left(a_{2}-a_{2}^{\prime}\right)(2 \pi)^{3} .
\end{aligned}
$$

Thus if, on making the transition from a discrete to a continuous basis we interpret $\sum_{i}$ as

$$
\sum_{i} \equiv \int_{0}^{\infty} d x \int_{-\infty}^{+\infty} d a_{2} \int_{-\infty}^{+\infty} d a_{2}(2 \pi)^{-3} c^{-1}
$$

then for each chart the set $\left\{P_{i}, n_{i}\right\}$ from a basis which is clearly complete since it reduces to simple exponentials on surfaces of constant $U$. Note that the basis breaks down when the chart breaks down. This is due to the same focussing effect which acts on timelike and null geodesics and the elementary waves making up the basis. These begin in $\mathscr{M}^{-}$or $\mathscr{M}^{+}$as ordinary plane waves in Minkowski space (they thus describe real particles in $\mathscr{M}^{-}$and $\mathscr{M}^{+}$) but as $a(u) \rightarrow 0$ they become focussed, the phase runs through an infinite amount and their amplitude diverges. It is instructive to compare the wave and particle aspects of this purely classical problem. In general if we attempt to solve the Klein-Gordon equation with the ansatz

$$
\psi=A \exp i S, \quad A, \quad S \quad \text { real }
$$

we obtain

$$
\left(\square^{2} A\right) / A=(\nabla S)^{2}-m^{2} .
$$

The W.K.B. approximation consists of letting $S$ satisfy the Hamilton-Jacobi equation $(\nabla S)^{2}=m^{2}$. From this is follows that $A^{-2}$ is the determinant of the three metric orthogonal to the geodesics whose normals are $\nabla S$. In this case the approximation is exact. Thus the waves, the co-ordinate system and the geodesics associated with them all focus on the same surface.

Now if we express three solutions in harmonic co-ordinates we find the $V$ dependence is of the form expicV. Since in harmonic co-ordinates

$$
(\phi, \psi)=(2 i)^{-\frac{1}{2}} \iint(\bar{\Phi} \partial \psi / \partial V-\psi \partial \bar{\Phi} / \partial V) d V d X^{1} d X^{2}
$$

we see that only solutions with the same $c$ (regardless of the form of $a_{i j}(u)$ ) fail to be orthogonal. Thus the coefficients $\beta_{i j}^{(+)}$and $\beta_{i j}^{(-)}$connecting any two bases (defined by their corresponding charts) vanish. In particular the $\beta$ 's relating $B$ and $B_{+}$will vanish if the bases and charts have a common region of validity. If the domains of validity do not overlap it is necessary to introduce intermediate charts and bases and compute the $\beta$ 's in stages. This works because the Bogoliubov transformations form a group. The final result is the same: no matter how strong the wave-no particle creation takes place.

We now turn to the calculation of propagators. Since the various bases are related by non-mixing Bogoliubov transformations there is no ambiguity and we 
may chose any convenient basis to work in. We begin by calculating:

$$
\begin{aligned}
& \mathscr{G}^{(+)}\left(x, x^{\prime}\right)=i \sum_{i} p_{i}(x) \bar{p}_{i}\left(x^{\prime}\right) \\
& =i \int_{0}^{d} d c \int_{-\infty}^{+\infty} d a_{1} \int_{-\infty}^{+\infty} d a_{2} \\
& \cdot(2 \pi)^{-3} c^{-1} \exp i\left\{a_{i}\left(x^{i}-x^{\prime i}\right)+\left[c\left(v-v^{\prime}\right)+\left(u-u^{\prime}\right) B^{i j}\left(u, u^{\prime}\right) a_{i} a_{j}\right] / 4 c\right\} .
\end{aligned}
$$

A straight forward calculation leads to:

$$
\mathscr{G}^{(+)}=-\left(16 \pi^{2}\right)^{-1}\left(a(u) a\left(u^{\prime}\right)\right)^{-\frac{1}{2}} \operatorname{det}\left|B_{i j}\left(u, u^{\prime}\right)\right| \int_{0}^{\infty}\left(u-u^{\prime}\right) \exp i\left(m^{2} / s+\Omega s / 2\right) d s
$$

with $s=4 c\left(u-u^{\prime}\right)^{-1}$.

To obtain $\mathscr{G}^{(-)}$we must integrate over negative $c$ but since

$$
\mathscr{G}_{F}=\begin{array}{ll}
\mathscr{G}^{(+)} & u>u^{\prime} \\
\mathscr{G}^{(-)} & u<u^{\prime} .
\end{array}
$$

$S$ remains the same. The order of the limits of integration is, however, the opposite from that in (3.9) thus we obtain

$$
\begin{aligned}
\mathscr{G}_{F} & =-\left(16 \pi^{2}\right)^{-1} \Delta^{\frac{1}{2}} \int_{0}^{\infty} \exp i\left(m^{2} / s+\Omega s / 2\right) d s \\
& =-\left(16 \pi^{2}\right)^{-1} \Delta^{\frac{1}{2}} \int_{0}^{\infty} t^{-2} \exp i\left(m^{2} t+\Omega / 2 t\right) d t .
\end{aligned}
$$

We may set $m^{2}=0$ in 3.9 and using the definitions in Section 2 obtain:

$$
\overline{\mathscr{G}}\left(x, x^{\prime}\right)=-(8 \pi)^{-1} \delta(\Omega) \text {. }
$$

This shows that the massless wave equation propagates on $\mathscr{M}$ without back scattering or tails. That is Huyghen's Principle is obeyed. That this is true for plane-waves seems well known. McClenaghen has proved a partial converse the only vacuum spacetimes for which this is true are plane waves (16)]. If $\mathrm{m}^{2}=0$ there will be tails but they will have the same Hankel Function form as in flat space. Friedlander [17] and Zauderer [18] have obtained an essentially equivalent result.

We may now use the limiting values of $\Delta_{\mu}, \Delta_{\mu^{\prime}}, v$ and $\Omega_{\mu}$ which are zero and that of $\Omega_{\mu v^{\prime}}$ which is $g_{\mu \nu}$ to show that, if we take these limits before performing the integral we obtain a divergent multiple $\Lambda$ of $g_{\mu \nu}$ as $\left\langle 0_{-}\left|\boldsymbol{T}_{\mu \nu}\right| 0_{-}\right\rangle$. By similar reasoning we find that using (1.14) we obtain the same answer - placing $\sqrt{\Delta}\left(x, x^{\prime}\right)$ in front of the flat space propagator does not alter $\left\langle 0_{-}\left|\boldsymbol{T}_{\mu \nu}\right| 0_{-}\right\rangle$. This divergence could be absorbed into Einstein's equations with cosmological constant.

$$
G_{\mu \nu}=8 \pi\left\langle 0\left|\boldsymbol{T}_{\mu \nu}\right| 0\right\rangle-\lambda g_{\mu \nu}
$$

by an infinite renormalization of the cosmological constant $\lambda$. In order to remain a plane wave spacetime $\Lambda$ and $\lambda$ must precisely cancel. However, since these limits are not very well defined it would seem more reasonable to adopt a PauliVillars scheme and regularize the propagator by subtracting from it (1.15). This will give zero and hence a zero answer for $\left\langle 0_{-}\left|\boldsymbol{T}_{\mu \nu}\right| 0_{-}\right\rangle$.

Adiabatic regularization on the other hand could presumably be achieved by subtracting from $\mathscr{G}_{F}\left(x, x^{\prime}\right)$ the quantity

$$
A\left(x, x^{\prime}\right)=-\left(16 \pi^{2}\right)^{-1} \int_{0}^{\infty} \exp i\left\{n^{2} / s+\left(U-U^{\prime}\right)\left(V-V^{\prime}\right) s / 2-X^{i} X^{\prime} s / 2\right\} d s .
$$


However, this would give a combination of terms some proportional to $g_{\mu \nu}$ and some to $\eta_{\mu v}$, the flat space metric. One would not expect them to cancel. This is because adiabatic regularization makes use of - implicity at least - two different light cones. For this reason we shall not use adiabatic regularization.

From these remarks it would seem that the only reasonable value for $\left\langle 0_{-}\left|\boldsymbol{T}_{\mu \nu}\right| 0_{-}\right\rangle$in this spacetime is zero. This would mean that a gravitational wave can propagate through spacetime unhindered by quantum effects - however strong it is.

These results may be understood in three ways:

(1) The absence of scalar invariants, upon which quantum effects must presumably depend means that on such effects must be absent.

(2) The existence of a global killing vector - in this case the null vector $\partial / \partial V$ against which "frequencies" are being measured means that no mixing takes place just as it would if $\mathscr{M}$ were globally static.

(3) The absence of back scattering precludes particle creation. Indeed since a "graviton" presumably in some sense carries lightlike momentum the creation of one or more particles with timelike or lightlike momentum would violate the conservation of momentum unless the created particles were massless and precisely aligned with the momentum of the graviton. This is presumably also the physical reason why back scattering does not occur in plane wave spacetimes cf. [19].

\section{The DeWitt-Schwinger Approach}

DeWitt, following methods pioneered by Schwinger for quantum electrodynamics, has obtained some general results which it is of interest to compare with the particular discussion given above. He considers a spacetime with two flat regions in the infinite past and infinite future as we have done. He constructs the quantity

$$
H\left(x, x^{\prime}\right)=i \int_{0}^{\infty} \psi\left(x, x^{\prime}, t\right) d t
$$

where $\psi$ obeys

$$
\begin{aligned}
\square^{2} \psi+m^{2} \psi & =i \partial \psi / \partial t, \\
\psi\left(x, x^{\prime}, 0\right) & =\delta\left(x, x^{\prime}\right) .
\end{aligned}
$$

As a solution of (4.2) he takes

$$
\psi=-\left(16 \pi^{2}\right)^{-1} \Delta^{\frac{1}{2}} t^{-2} \exp i\left\{m^{2} t+\Omega / 2 t\right\} \sum_{n=0}^{n=\infty} a_{n}(i t)^{n}
$$

with $a_{0}=1$ and the $a_{n}\left(x, x^{\prime}\right)$ to obey the recurrence relations:

$$
\Omega^{\mu} a_{n+1, \mu}+(n+1) a_{n+1}=\Delta^{-\frac{1}{2}}\left(\Delta^{\frac{1}{2}} a_{n}\right)_{; \mu}^{\mu}
$$

deWitt then claims that $H\left(x, x^{\prime}\right)$ which obeys the equation

$$
\square \square^{2} H+m^{2} H=\delta\left(x, x^{\prime}\right)
$$

can be identified as

$$
\mathscr{G}_{\infty}\left(x, x^{\prime}\right)=i\left\langle 0_{+}\right| T\left\{\phi(x) \phi^{\dagger}\left(x^{\prime}\right)\left|0_{-}\right\rangle /\left\langle 0_{+} \mid 0_{-}\right\rangle\right.
$$


since in the flat regions where $a_{n}->0 n>0$ (4.4) coincides with the flat space Feynman propagator and so will possess the property of propagating positive frequences to the asymptotic future and negative frequencies to the asymptotic past. It is possible to relate the $a_{n}$ 's directly to the coefficients of the Hadamard series for the Green's functions of the massless wave equation and in particular the vanishing of all $a_{n} ; n>0$ will ensure that Huyghen's principle will hold for that Eqs. $[11,13]$.

Then defining

$$
\left\langle 0_{+} \mid 0_{-}\right\rangle=\exp i W
$$

he obtains the equations

$$
\begin{aligned}
\left\langle 0_{+}\left|T_{\mu \nu}\right| 0_{-}\right\rangle\left\langle 0_{+} \mid 0_{-}\right\rangle & =\delta W / \delta g^{\mu \nu} \\
W & =\int L_{\text {eff }} \sqrt{-g} d^{4} x \\
L_{\text {eff }} & =\operatorname{Lt}_{x \rightarrow x^{\prime}} \int_{0}^{\infty} t^{-1} \psi\left(x, x^{\prime}\right) d t,
\end{aligned}
$$

where $\delta / \delta g^{\mu v}$ denotes functional differentiation.

(4.8) is a fairly straight forward consequence of the Schwinger Action Principle which can be proved from the theory we have developed so far but (4.9) appears to make essential use of somewhat symbolic manipulations of "continuous matrixes". The reader is referred to de Witt's article for the details.

The $L_{\text {eff }}$ so obtained is of the form

$$
\begin{aligned}
L_{\mathrm{eff}}= & \alpha_{0}+\alpha_{1} R / b+\alpha_{3}\left\{R_{i \mu}^{i \mu}+R^{2} / 72-R_{\alpha \beta} R^{\alpha \beta} / 180\right\}, \\
& +\sum_{n=3}^{n=\infty} \alpha_{n} m^{-2 n}{\underset{x t}{L \rightarrow x^{\prime}}}_{n} a_{n}\left(x, x^{\prime}\right)
\end{aligned}
$$

where $\alpha_{0}, \alpha_{1}, \alpha_{2}$ are numbers depending on $m^{2}$ which if the limits in (4.9) are taken diverge and the $\alpha_{n}, n \geqq 3$ are finite numbers. In the plane wave case, since the $\operatorname{Lt}_{x \rightarrow x^{\prime}} a_{n}\left(x, x^{\prime}\right)$ can involve only invariants of $g_{\mu v}, R_{\mu v \sigma \varrho}$ and its derivatives, we are left with only the first term - which would occur in flat space. Thus the results obtained in Sections 2 and 3 are entirely consistent with de Witt's analysis.

In the more general case de Witt's arguments show that the divergent parts of $L_{\text {eff }}$ are of a more complicated structure than we have found. If one seeks to eliminate them by adding "counter terms" to the Einstein Lagrangian one will need terms of a different form from those already present. If this is done in such a way as to eliminate the first three terms then it is $a_{3}\left(x, x^{\prime}\right)$ which will give the lowest order correction to $\left\langle 0_{+}\left|\boldsymbol{T}_{\mu \nu}\right| 0_{-}\right\rangle$. However, even if we were to calculate this it would not give $\left\langle 0_{-}\left|\boldsymbol{T}_{\mu \nu}\right| 0_{-}\right\rangle$since in general $\left|0_{+}\right\rangle \neq\left|0_{-}\right\rangle$though it would work in a globally static, asymptotically flat spacetime.

\section{Conclusion}

In this paper we have shown that a plane gravitational wave will propagate without creating particles from the vacuum. This result will also be true of any gravitational wave far from its (bounded) source. If we adopt a fairly natural decision as to what to use for the vacuum expectation value of the energy 
momentum tensor it vanishes and the wave travels unimpeded. This analysis is entirely consistent with the work of de Witt which indicates that in more general cases the divergences of $\left\langle 0_{-}\left|\boldsymbol{T}_{\mu \nu}\right| 0_{-}\right\rangle$are more serious than we have encountered here. This absence of particle creation effects seems to stem entirely from the symmetry of the situation and makes no use of the Einstein equations. It is closely analogous to the situation for plane electromagnetic waves and it seems reasonable to suppose that the same results would hold for a combined gravitational-electromagnetic wave. In order to investigate the properties of $\left\langle 0_{-}\left|\boldsymbol{T}_{\mu \nu}\right| 0_{-}\right\rangle$in a more general context and especially the important case of black hole explosions more work will have to be done.

Acknowledgements. I should like to thank A. King for helpful discussions on plane waves and B. DeWitt for a stimulating discussion of his approach to these problems.

\section{References}

1. Hawking, S. W.: Nature 248, 30 (1974)

2. Hawking, S. W.: Commun. math. Phys. 43, 199-220 (1975)

3. Gibbons, G. W.: Commun. math. Phys. 44, 245-264 (1975)

4. Fulling, S., Parker, L.: Ann. Phys. 87, 176 (1974)

6. Ellis, G.F.R., Hawking, S. W.: The large scale structure of spacetime C.U.P. (1974)

7. Schwinger, J.: Phys. Rev. 82, 664 (1951)

8. Schwinger, J. : Proc. Nall. Acad. Sci. 37, 452 (1951)

9. Pauli, W., Villars, F.: Rev. Mod. Phys. 21, 434 (1949)

10. Zel'dovich, Ya.B., Starobinsky, A. A.: Sov. Phys. - J.E.T.P. 34, 1159 (1972)

11. DeWitt, B., Brehme, R.: Ann. Phys. 9, 220 (1960)

12. DeWitt, B., Utiyama, R.: J. Math. Phys. 3, 608 (1962)

13. DeWitt, B. : In: DeWitt, C., DeWitt, B. (Eds.): Relativity, groups, and topology. Blackie 1964

14. Ehlers, J., Kundt, W.: In: Witten, L.(Ed.): Gravitation. Chichester: Wiley 1962

15. Penrose, R.: Rev. Mod. Phys. 37, 215 (1965)

16. McClenaghen, R.: Proc. Cam. Phil. Soc. 65, 139 (1969)

17. Friedlander, G.: Second order hyperbolic differential equations. C.U.P. (to be published)

18. Zauderer,E.: J. Inst. Math. Appl. 8, 8 (1971)

19. Rosen, G.: Phys. Rev. 128, 449 (1962)

Communicated by J. Ehlers

Received November 13, 1974 